\title{
SLC7A5 act as a potential leukemic transformation target gene in myelodysplastic syndrome
}

\author{
Yan Ma ${ }^{1}$, Jing Song ${ }^{1}$, Bobin Chen ${ }^{1}$, Xiaoping $\mathrm{Xu}^{1}{ }^{1}$, Guowei Lin ${ }^{1}$ \\ ${ }^{1}$ Department of Hematology, Huashan Hospital, Fudan University, Shanghai, 200040, China \\ Correspondence to: Xiaoping XU, e-mail: xpxU1111@163.com \\ Keywords: myelodysplastic syndrome, miRNA, SLC7A5 gene, SKM- 1 cell line, siRNA \\ Received: July 22, $2015 \quad$ Accepted: November 21, $2015 \quad$ Published: December 09, 2015
}

\section{ABSTRACT}

Objective: Myelodysplastic syndromes (MDS) are a heterogenous group of clonal hematopoietic stem cell disorders characterized by increased risk of leukemic transformation. This study identifies microRNAs(miRNA) and miRNA targets that might represent leukemic transformation markers for MDS.

Methods: Based on our previously established nested case-control study cohort of MDS patients, we chose paired patients to undergo Angilent $8 \times 15 \mathrm{~K}$ human miRNA microarrays. Target prediction analysis was administrated using targetscan 5.1 software. We further investigated the function of target gene in MDS cell line using siRNA method, including cell proliferation, cell apoptosis, cell cycle and electron microscope.

Results: Finally we screened a subset of 7 miRNAs to be significantly differentially expressed between the case (at the end of follow up with leukemic transformation) and control group (at the end of follow up without leukemic transformation). Target prediction analysis revealed SLC7A5 was the common target gene of these 7 miRNAs. Further study on the function of SLC7A5 gene in SKM-1 cell line showed that downregulation of SLC7A5 inhibited SKM-1 cells proliferation, increased apoptosis and caused cell cycle arrest in the G0/G1 stage.

Conclusion: Our data indicate that SLC7A5 gene may act as a potential leukemic transformation target gene in MDS.

\section{INTRODUCTION}

Myelodysplastic syndrome (MDS) are a heterogenous group of clonal hematopoietic stem cell disorders characterized by ineffective and dysplastic hematopoiesis and increased risk of leukemic transformation [1-4]. Patients evolving into leukemia respond poorly to chemotherapy and die within a short time. Hence, it is very important to find detectable marker to predict the probability of leukemic transformation once MDS has been diagnosed. MiRNAs (miRNA) are small regulatory, non-coding RNAs. It is believed that miRNAs primarily affect the stability of mRNA and/or the initiation and progression of protein translation [5-8]. Even though the biological function of miRNA is yet to be fully understood, it has been shown that miRNAs play multiple roles in cancer genesis, development, invasion and metastasis [9-12]. Compared with mRNA, miRNAs are more stable and specific so as to be an ideal tumor marker. However, there are few studies on miRNAs involved in MDS transformation [13-15].

Based on our previous study, we established a nested case-control cohort of MDS patients [16, 17]. At the end of the follow-up, patients progressed into leukemia were classified as the case group, whereas patients without leukemic transformation were classified as the control group. In this study, we studied the miRNAs profiling of bone marrow monoclonal cells of MDS patients in the case and control groups. We finally screened a subset of 7 miRNAs to distinguish the case and control group remarkably. By predicting target gene of above seven miRNAs using targetscan 5.1 software, we found that SLC7A5 gene is the common target gene of above7 miRNAs. As SLC7A5 gene is one of the leukemic transformation associated gene resulted from our previous study [17], we further investigated the function of SLC7A5 gene in MDS cell line by downregulating the expression of SLC7A5 gene using siRNA method. 
Table 1: The clinical characteristics of paired patient for miRNA microarray assay

\begin{tabular}{|c|c|c|c|c|}
\hline Group & WHO subtype & Gender & Age & Cytogenetcis \\
\hline case 1 & RCMD & female & 26 & $46, \mathrm{XX}[20]$ \\
\hline case 2 & RCMD & male & 48 & $46, X Y[20]$ \\
\hline case 3 & RCMD & female & 77 & $45, X,-X[16] / 46, X X[4]$ \\
\hline case 4 & RCMD & male & 49 & $46, X Y[20]$ \\
\hline case 5 & RCMD & male & 62 & $46, X Y[20]$ \\
\hline case 6 & RAEB-1 & male & 70 & $\begin{array}{c}\text { 46,XY [10]/46,XY,del(5)(q31q35),t(10;11)(p13;q23),add(18)(p11.2) } \\
{[6] / 47, \text { idem, }+19[2] / 46, \operatorname{idem}, \operatorname{del}(1)(\mathrm{p} 22), \operatorname{der}(6) \mathrm{t}(1 ; 6)(\mathrm{p} 32 ; \mathrm{q} 22), \operatorname{der}(10)} \\
\mathrm{t}(10 ; 11) \operatorname{add}(10)(\mathrm{q} 24),+\operatorname{del}(14)(\mathrm{q} 24)[2]\end{array}$ \\
\hline case 7 & RAEB-1 & female & 73 & $46, \mathrm{XX}[2] / 46, \mathrm{XX}, \operatorname{del}(20)(\mathrm{q} 11.2 \mathrm{q} 12)[70] / 92$, idemx2 [3] \\
\hline case 8 & RAEB-1 & male & 77 & $46, X Y[5]$ \\
\hline case 9 & RAEB-2 & female & 56 & $46, \mathrm{XX}[20]$ \\
\hline case 10 & RAEB-2 & female & 55 & $46, X Y, \operatorname{del}(20)(q 11.2 q 12)[20]$ \\
\hline control 1 & RCMD & female & 32 & $46, X X[20]$ \\
\hline control 2 & RCMD & male & 42 & $46, X Y, \operatorname{del}(20)(q 11.2 q 12)[20]$ \\
\hline control 3 & RCMD & female & 76 & $46, \mathrm{XX}, \mathrm{t}(1 ; 3)(\mathrm{p} 36.1 ; \mathrm{q} 21)[19] / 46, \mathrm{XY}[1]$ \\
\hline control 4 & RCMD & male & 48 & $46, X Y[20]$ \\
\hline control 5 & RCMD & male & 69 & $46, X Y[20]$ \\
\hline control 6 & RAEB-1 & male & 68 & $\begin{array}{l}\text { 44,XY,der(5)t(5;11)(q13;q24),der(11)t(5;11)(?;q24),-18, } \\
\operatorname{add}(20)(\mathrm{q} 11.2),-21, \operatorname{idic}(22)(\mathrm{p} 11.2)[15] / 88, \operatorname{idemx} 2[4] / 46, \mathrm{XY}[1]\end{array}$ \\
\hline control 7 & RAEB-1 & female & 70 & 44,XX,dic(2;4)(q34;p16),-6,add(11)(q23) [15]/46,XX [5] \\
\hline control 8 & RAEB-1 & male & 69 & $46, X Y[6]$ \\
\hline control 9 & RAEB-2 & female & 50 & $46, \mathrm{XX}[20]$ \\
\hline control 10 & RAEB-2 & female & 53 & $46, X Y[20]$ \\
\hline
\end{tabular}

RCMD = Refractory Cytopenia with Multilineage Dysplasia; RAEB = Refractory Anemia with Excess of Blasts

\section{RESULTS}

\section{miRNA spectra of the MDS patients in the case and control groups}

From the above nested case-control study cohort of the MDS patients, paired patients were chosen for the gene expression microarray test ( case $=10$, control $=10$ ) according to age, gender, WHO subtype, and IPSS cytogenetic subgroup, which are risk factors of leukemic progression in MDS patients (Table 1). We adapted a microarray platform from Agilent to profile the miRNA spectra. Excluding any miRNA with hybridization intensity $<1.5$ times the global mean intensity, there were20 miRNAs to be significantly differentially expressed between the case and control group, including 17 downregulated miRNA and 3 upregulated miRNA (Table 2). Cluster analysis of above 20 miRNAs resulted in a subset of 7 differentiall expressed miRNAs (Figure 1). By predicting target gene of above seven miRNAs using targetscan 5.1 software, we found that SLC7A5 gene is the common target genes of above 7 miRNAs. (Table 3), which was a MDS leukemic progression associated gene revealed in our previous study [17].

\section{Down regulation of SLC7A5 inhibits proliferation of SKM-1 cell line}

Image Master Total Lab Image system analysis showed SLC7A5 expression in SLC7A5-siRNAgroup, negative control and blank group was $1662.63 \pm 13.00$, $4529.63 \pm 24.36$, and $6653.03 \pm 18.76$ respectively, indicating the success of down-regulation of SLC7A5 by SLC7A5-siRNA (Figure 2). To further study the function of SLC7A5 gene, SKM-1 cell line was investigated by down-regulating the expression of SLC7A5 gene using siRNA method. Cell proliferation rates were measured by CCK-8 assay. The growth of cells inSLC7A5 downregulation group by SLC7A5 specific siRNA was significantly slower than that of the negative control group 
Table 2: Detectable differential miRNAs in the case and control patient group by microarray

\begin{tabular}{|c|c|c|c|}
\hline & miRNA & Fold change between Case vs. control & Expression in case group \\
\hline 1 & hsa-mir-1290 & 0.26 & down \\
\hline 2 & hsa-mir-342-5p & 0.22 & down \\
\hline 3 & hsa-mir-1224-5p & 0.23 & down \\
\hline 4 & hsa-mir-345 & 0.38 & down \\
\hline 5 & hsa-mir-1228 & 0.38 & down \\
\hline 6 & hsa-mir-1249 & 0.32 & down \\
\hline 7 & hsa-mir-1826 & 0.26 & down \\
\hline 8 & hsa-miR-1306 & 0.38 & down \\
\hline 9 & hsa-miR-188-5p & 0.43 & down \\
\hline 10 & hsa-miR-320a & 0.48 & down \\
\hline 11 & hsa-miR-320c & 0.26 & down \\
\hline 12 & hsa-miR-365 & 0.31 & down \\
\hline 13 & hsa-miR-423-5p & 0.35 & down \\
\hline 14 & hsa-miR-483-5p & 0.25 & down \\
\hline 15 & hsa-miR-634 & 0.31 & down \\
\hline 16 & hsa-miR-671-5p & 0.23 & down \\
\hline 17 & hsa-miR-939 & 0.24 & down \\
\hline 18 & hsa-miR-1246 & 2.22 & up \\
\hline 19 & hsa-miR-150 & 10.41 & up \\
\hline 20 & hsa-miR-574-5p & 8.04 & up \\
\hline
\end{tabular}

(Figure 3), demonstrating that down-regulation of SLC7A5 expression led to inhibition of growth of SKM-1 cells.

\section{Down regulation of SLC7A5 increases apoptosis of SKM-1 cell line}

Cell apoptosis was assayed by flow cytometry. The percentages of early apoptosis cells in SLC7A5-siRNA group increased significantly compared with negative control group $(38.9 \% \pm 3.6 \% \mathrm{Vs} 2.9 \% \pm 0.4 \%)$. Also, the percentages oflater apoptosis cells in SLC7A5-siRNA group increased significantly $(8.8 \% \pm 0.4 \%$ Vs $0.4 \% \pm$ $0.06 \%$ ) compared with negative control group (Figure 4). Those outcomes revealed that downregulation of SLC7A5 promoted apoptosis.

\section{Down regulation of SLC7A5 caused cell cycle arrest in the G0/G1stage}

The cell cycle analysis by flow cytometry illustrated SLC7A5-siRNA group cells in G0/G1 phase increased by $14 \%$ and cells in $\mathrm{S}$ phase reduced by $13 \%$ compared with negative control group cells (Table 4), (Figure 5) suggesting that cells of SLC7A5-siRNA group were obstructed at $\mathrm{G} 0 / \mathrm{G} 1$ phase.

\section{Electron microscopy}

We also observed the ultrastructural changes in response to the down-regulation of SLC7A5in SKM1 cells using electron microscopy. The SKM-1 cells of the control group were rich in mitochondria, which were normal in shape and size and had few lipid droplets (Figure 6A). In contrast, many mitochondria from SKM1 cells with transfection were enlarged and showed morphological changes, including a less dense matrix, a large lipid droplet, mitochondrial swelling, condensed cytoplasm, and karyopyknosis (Figure 6B).

\section{DISCUSSION}

To date, the prediction of the leukemic evolution of MDS is very difficult. Hence, a set of molecular markers enabling better prediction of disease progression 
and prognosis would be valuable. MiRNAs are small regulatory, noncoding RNAs and play multiple roles in cancer genesis, development, invasion and metastasis. Compared with mRNA, miRNAs are more stable and specific so as to be an ideal tumor marker. However, there are few studies on miRNAs involved in MDS transformation. Pons et al. detected miRNA expression in $25 \mathrm{MDS}$ patients (high risk group $=3$, low risk group $=22$ ) and found that miR-155, miR-181a and miR-222 were upregulated in high risk patients [13]. Hussein et al. studied miRNA expression of 24 MDS patients (high-risk group $=6$, low risk group $=18$ ) and found that MDS patients with different cytogenetic abnormalities had different miRNA expression profiles. And they showed miR-221 (not miR-222) was downregulated (not upregulated) in high-risk MDS patients [14]. Choi et al. investigated the involvement of miRNAs encoded by chromosomes 8 and $1 \mathrm{q}$ in 65 MDS patients. They found a significant upregulation of miR-194-5p and miR-320a in MDS patients compared with controls. They further found that miR-194-5p is a candidate diagnostic biomarker for MDS and that low miR-194-5p expression could be associated with poor overall survival for MDS patients [15]. In order to avoid the selection bias and improve the efficiency of miRNA microarray assay, our study carefully chose case and controls from our previously established nested case-control study cohort for miRNA microarray

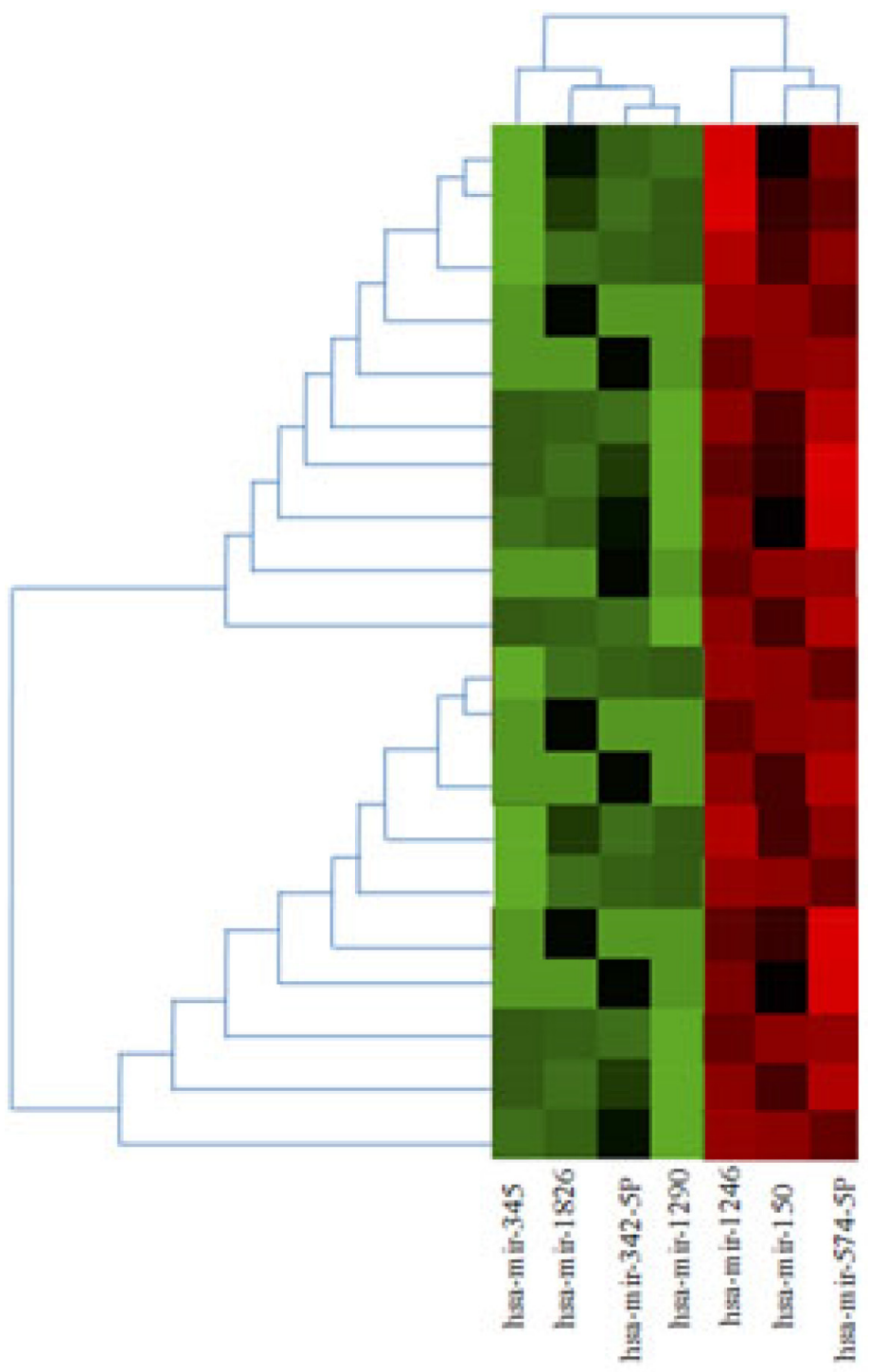

Figure 1: Heatmap and cluster analysis of miRNA expression in case and control group of MDS. We selected miRNAs with $p$ values of expressional differences less than 0.05 and/or $>2$-fold change between case and control group in miRNA array analysis. The heatmap generated by using heatmap software shows changes in the expression of these miRNAs in case group compared with controls. miRNAs whose expression is higher in case group are shown in red and those that are lower in green. The color key represents the values that are scaled to have a mean of zero and standard deviation of one. The identities of miRNAs are listed on the bottom, whereas the sample names are on the right. 
Table 3: Prediction of target gene of 7 miRNAs screened by miRNAmicroassay

\begin{tabular}{|c|c|}
\hline MiRNAs & Target Gene Symbol \\
\hline hsa-miR-345 & PUM2, PPP2R3A, BCAT1, ZFHX4, CHSY3, ARNT, SHE, SLC7A5, SOS1, ..... \\
\hline hsa-miR-1826 & SP1, CSNK1G1, AMD1, SLC37A2, SLC7A5, PKD1, WNT4, XPO1, ELFN2, C16orf72, ...... \\
\hline hsa-miR-342-5p & AFF4, GSG1L, CACNB1, NPTXR, C1QTNF3, ITGA10, ATXN7L3, SLC7A5, ...... \\
\hline hsa-miR-1290 & FOXN2, RSPO3, ZFP91, SLC39A9, PHF3, SLC12A6, STX6, TNFSF4, SLC7A5, ...... \\
\hline hsa-miR-1246 & $\begin{array}{c}\text { DMWD, C20orf27, FOXP4, CDC42SE1, BTBD14B,TMEM41A, GLP1R, } \\
\text { SRM,KLHL3,CCDC64,ENC1,SLC7A5, ...... }\end{array}$ \\
\hline hsa-miR-150 & HMP19,TBL1X,SRP9,PPARGC1A,ESRRG,LDR1,ZNF791,SLC7A5, ...... \\
\hline hsa-miR-574-5p & FOXP2,UBE2Q1,VGLL4,ARGLU1,YME1L1,CSDA,KCNMA1,_SLC7A5, ...... \\
\hline
\end{tabular}

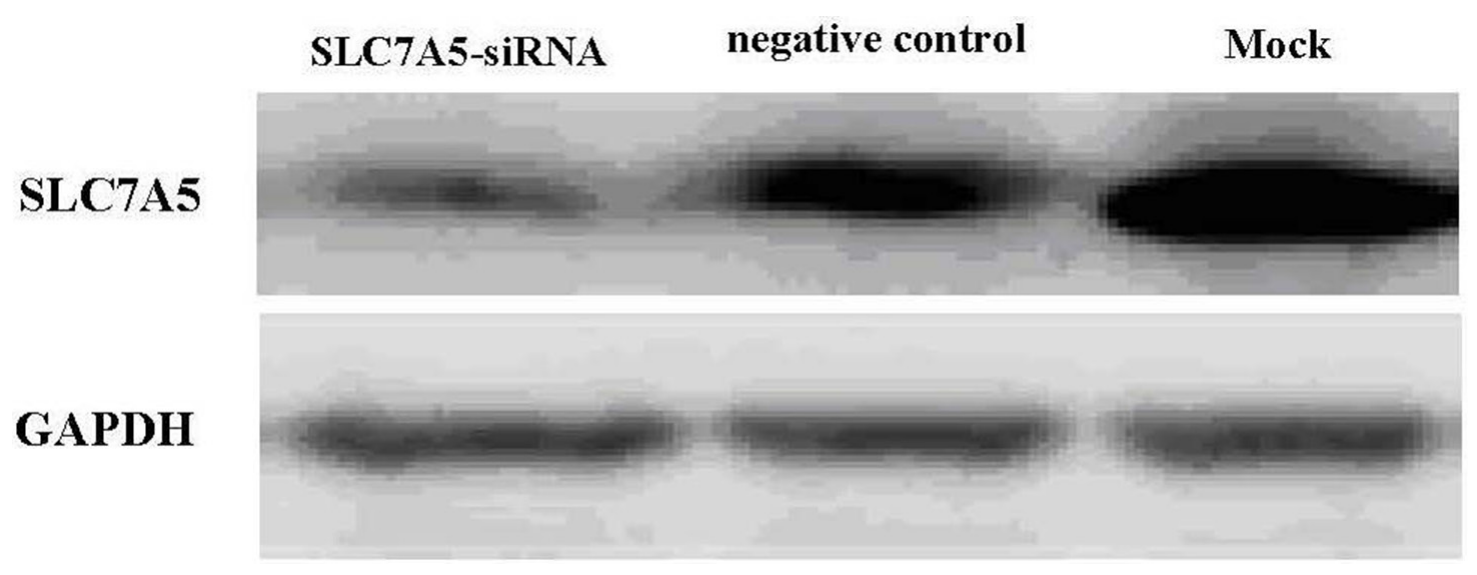

Figure 2: Western blot analysis of SLC7A5 protein. The protein expression was further confirmed by Western blot analysis after three days of transfection. The levels of the SLC7A5 protein were significantly lower in SLC7A5-siRNA group than in the negative control group and Mock. In comparison, GAPDH protein did not vary markedly among the three groups.

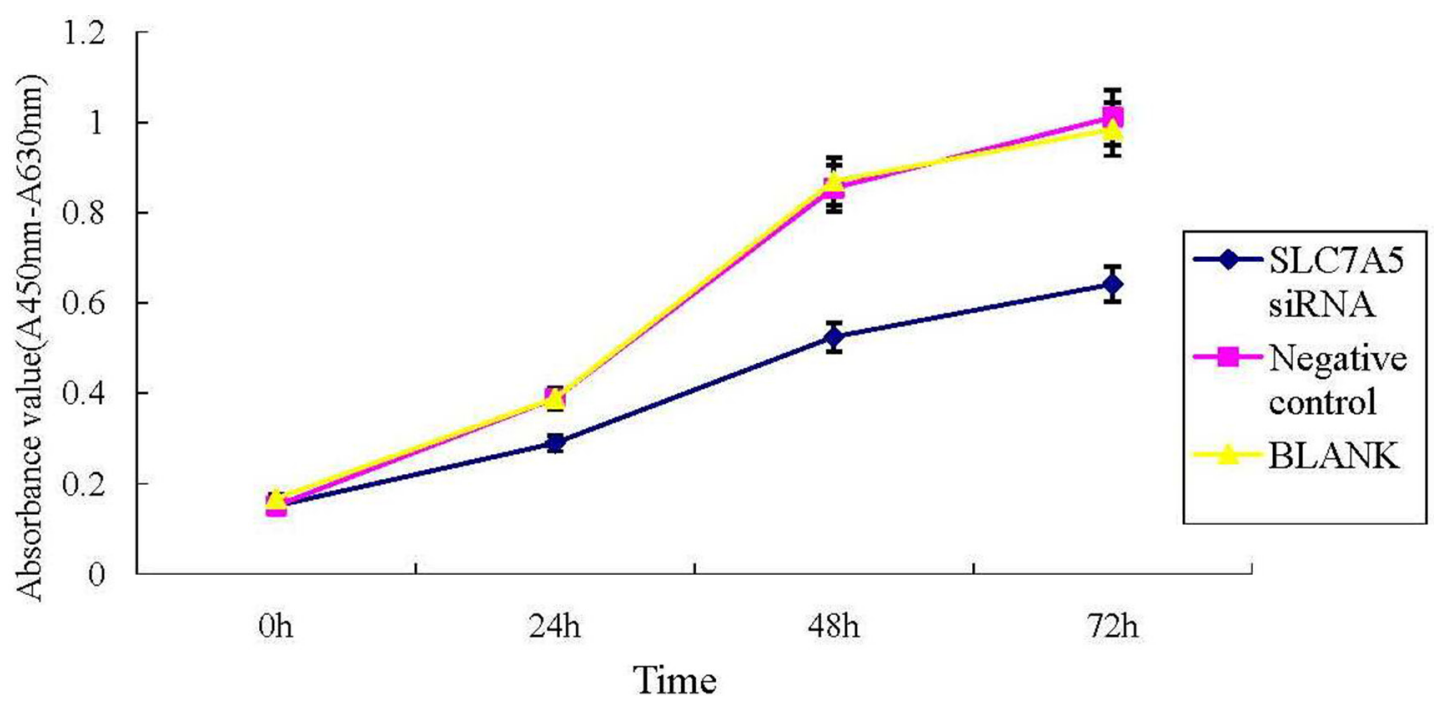

Figure 3: Down-regulation of SLC7A5 inhibits SKM-1 cells growth. Cell proliferation of SKM-1 cells using CCK-8 assays. The growth of SKM-1 cells in SLC7A5-siRNA group was significantly inhibited compared with the negative control (NC) group $(P<0.05)$. 
analysis according to the risk factors identified from the multivariate analysis so as to improve the efficiency of miRNA microarray. We identified 20 miRNAs to be significantly differentially expressed between the case and control group, including miR-320a which is coincident with Choi et al. Of above 20 miRNAs, there were 17 downregulated miRNA and 3 upregulated miRNA, which was coincident with our previous study showing more upregulated genes than downregulated genes in case group [17]. We finally screened a subset of 7 miRNAs to distinguish the case and control group remarkably, including 4 down-regulated miRNA and 3 up-regulated miRNA. By predicting target gene of above seven miRNAs using targetscan 5.1 software, we found that SLC7A5 gene is the common target genes of above 7 miRNAs.

SLC7A5 or LAT1 (L-type amino-acid transporter 1) encodes amino acid transporters and is involved in the transportation of essential amino acids. The action of SLC7A5 correlates with cell growth. A previous study revealed the upregulation of SLC7A5 in many human primary tumors possibly because of the constant, high demand for nutrients in tumor cells. This result indicates that SLC7A5 may be a potential target for tumor treatment

A

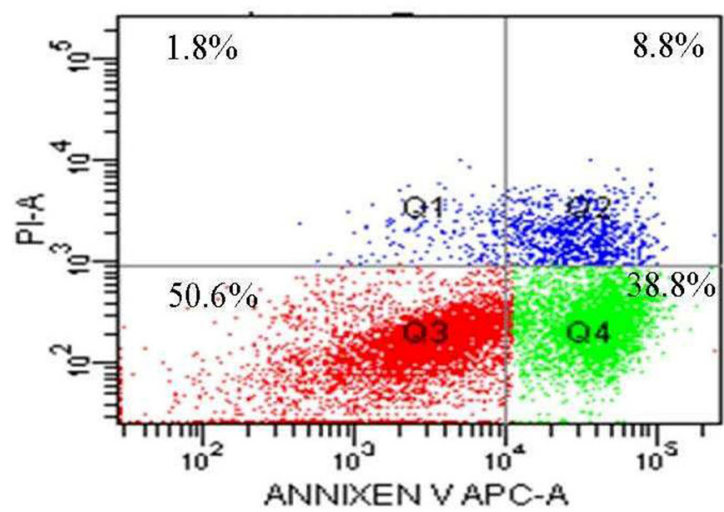

SLC7A5-siRNA

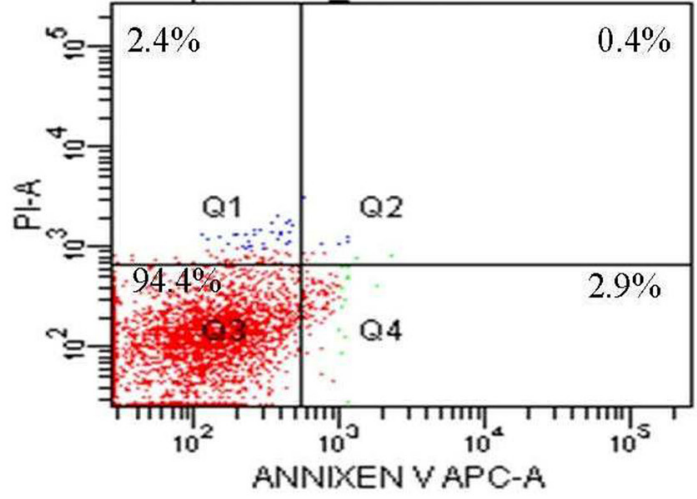

Negative Control

B

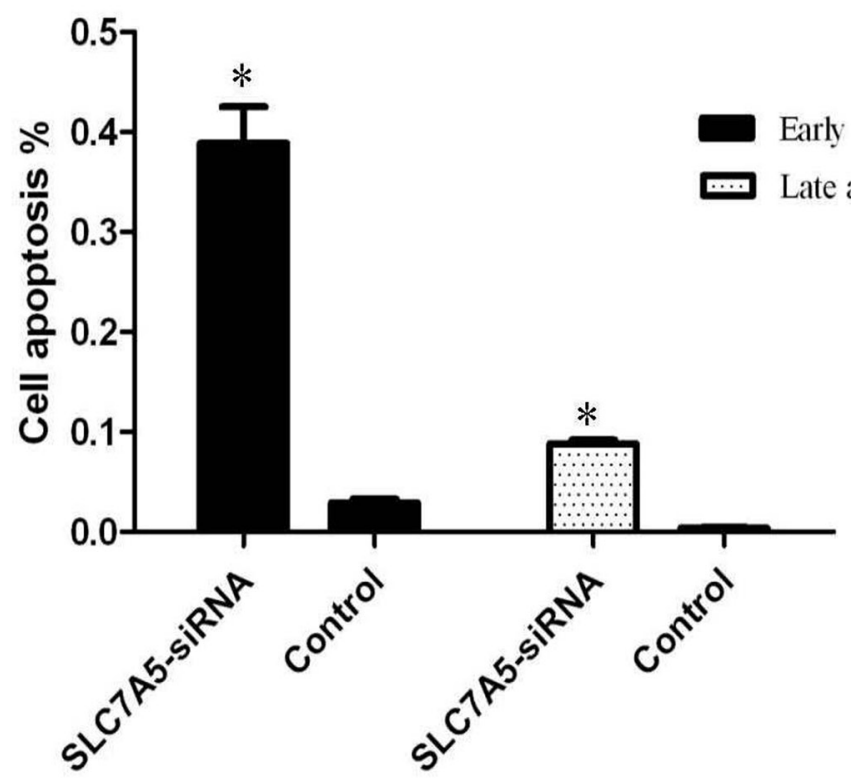

Figure 4: Down-regulation of SLC7A5 induces SKM-1 cells apoptosis. Cell apoptosis analysis of SLC7A5-siRNA group and negative control group cells was analyzed by flow cytometry. A. Representative histograms of annexin V/PI double-staining flow cytometry. B. Percent apoptosis (including early and late apoptotic cells) determined by flow cytometry ( $\mathrm{n}=3$ ). $* P<0.05$ vs control. 
Table 4: Cell cycle assay by flow cytometry in SLC7A5-siRNAand negative control group

\begin{tabular}{|l|c|c|c|}
\hline \multicolumn{2}{|c|}{ G1 phase (\%) } & S phase (\%) & G2 phase (\%) \\
\hline SLC7A5-siRNA & $54.55 \pm 1.16$ & $38.55 \pm 1.27$ & $6.90 \pm 0.13$ \\
\hline Pegative control value & $40.36 \pm 1.11$ & $52.33 \pm 0.73$ & $7.31 \pm 0.42$ \\
\hline
\end{tabular}
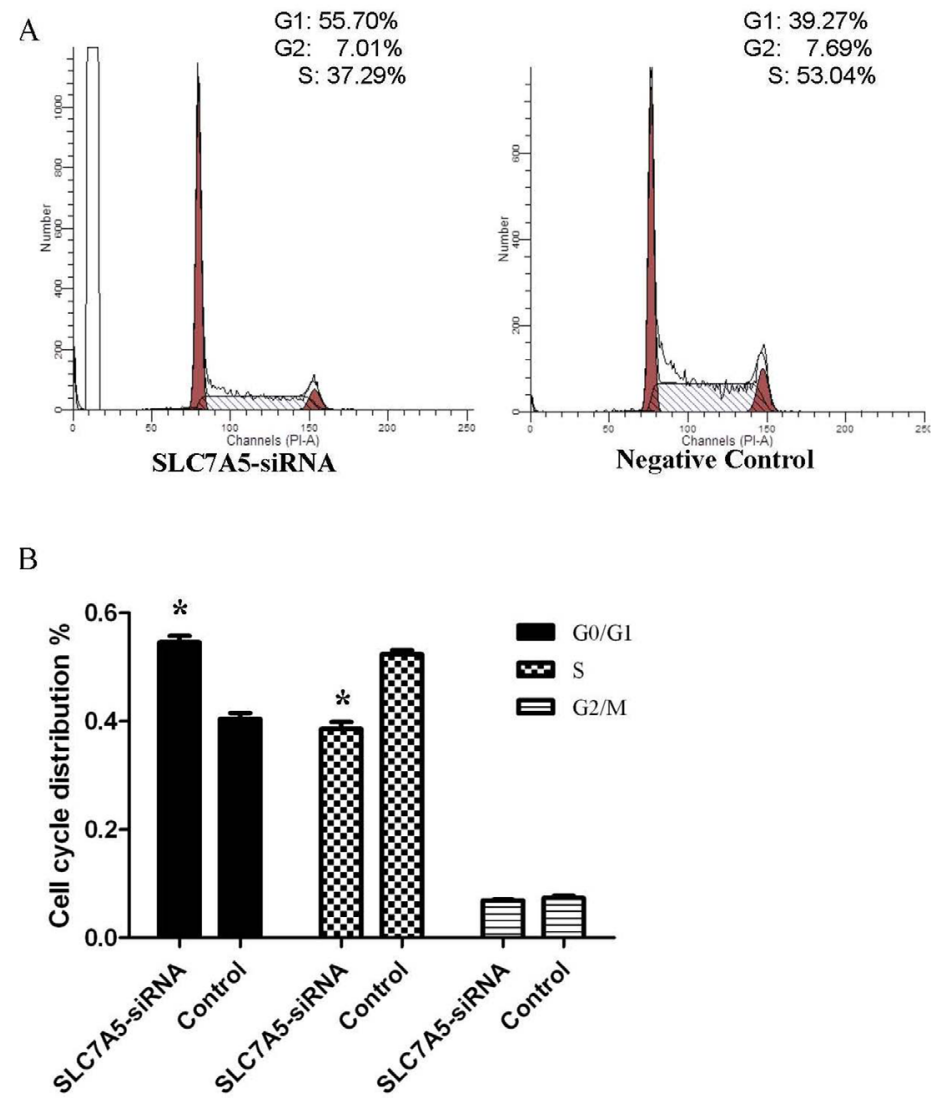

Figure 5: Down-regulation of SLC7A5 induces cell cycle arrest at G2/M phase in SKM-1 cells. Cell cycle distribution of SLC7A5-siRNA group and negative control group cells was analyzed by flow cytometry with PI staining after $48 \mathrm{~h}$ treatment. A. Representative flow cytometry histograms. B. Cell distribution at $\mathrm{G} 0 / \mathrm{G} 1, \mathrm{~S}$, and $\mathrm{G} 2 / \mathrm{M}$ phases of the cell cycle $(\mathrm{n}=3$ ). $* P<0.05$ vs control.

A

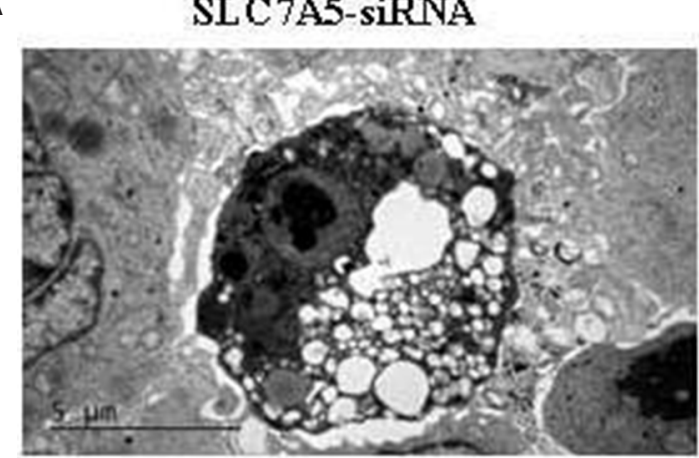

Negative Control

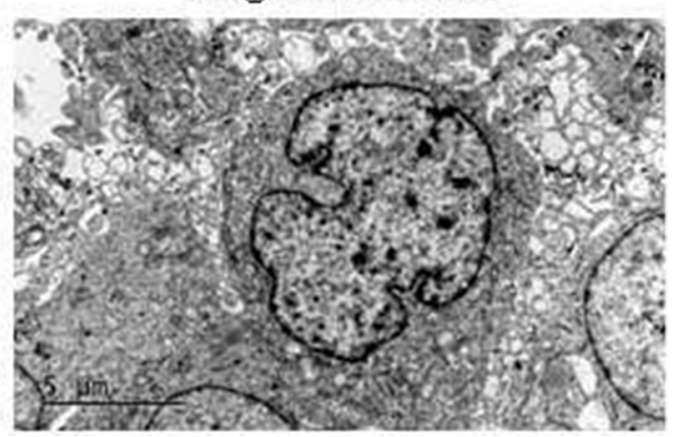

Figure 6: Down-regulation of SLC7A5 induces apoptosis in SKM-1 cells. Ultrastructural changes in SKM-1 cells. A. SKM-1 cells without transfection. B. SKM-1 cells with TUBB-RNAi transfection. (magnification 94,000). There are plenty of lipid droplet (arrow) and mitochondrial swelling in SKM-1 cells with TUBB-RNAi transfection. 
by downregulating the SLC7A5 expression to inhibit the proliferation of tumor cells [18-22]. Imai et al. reported the SLC7A5 overexpression in patients with stage I nonsmall-cell lung cancer (NSCLC), and the multivariate analysis showed that SLC7A5 expression is a promising pathological factor for the prognosis of patients with NSCLC [23]. Our previous study identified a subset of six genes associated with the evolution of MDS to acute leukemia, including SLC7A5 [17]. In order to further investigate the probable function of SLC7A5 in MDS, our present study on SLC7A5 gene in SKM-1 cell line showed that downregulation of SLC7A5 inhibited SKM-1 cells proliferation, increased apoptosis and caused cell cycle arrest in the G0/G1 stage, indicating that upregulation of SLC7A5 in MDS may increase the proliferation of malignant clones then accelerate the leukemic evolution.

In all, our study on MDS patients and MDS cell line strongly suggested that SLC7A5 gene may act as a potential leukemic transformation target gene in MDS, which will facilitate the prediction of MDS leukemic progression and may have clinical utility for the advanced identification of MDS patients with a propensity toward leukemia.

\section{MATERIALS AND METHODS}

\section{Patients and ethics statement}

Based on our nested case-control study cohort of MDS patients, we chose paired patients in 1:1 ratio according to risk factors for leukemic evolution. Case group included MDS patientswith leukemic transformation at the end of follow up and control group included MDS patients without leukemic transformation at the end of follow up. All patients gave their written informed consent. This study is in accordance with the modified Helsinki Declaration, and the protocols received approval from the ethics committees of the participating institutions (Institutional Review Board of Huashan Hospital, Fudan university).

\section{RNA extraction}

The total RNA from bone marrow mononuclear cells was extracted using TRIzol (Invitrogen, Paisley, U.K.) according to the manufacturer's protocol. Aliquots of the RNA samples were conserved for quality evaluation using Agilent 2100 Bioanalyzer (Agilent Technologies, Palo Alto, CA).

\section{Array hybridization, data analyses and target prediction of miRNAs}

To assess the level and composition of miRNA, miRNA arrays from Agilent Technologies were used. The RNA samples were labeled and processed according to manufacturer's recommended protocols. In brief, $\approx 100 \mathrm{ng}$ of total RNA was dephosphorylated with calf intestinal alkaline phosphatase, followed by denaturing with heat in the presence of dimethyl sulfoxide (DMSO). A cyanine dye, cyanine3-cytidine bisphosphate $(\mathrm{pCp})$, was then joined to the dephosphorylated single-stranded RNA (including miRNA) by T4RNA ligase. MicroBioSpin 6 columns (Bio-Rad) were used to remove any unincorporated cyanine dye from the samples. The purified labeled miRNA probes were hybridized to $8 \times 15 \mathrm{~K}$ human miRNA microarrays in a rotating hybridization oven at $20 \mathrm{rpm}$ for $20 \mathrm{~h}$ at $55^{\circ} \mathrm{C}$. After hybridization, the arrays were washed in Agilent gene expression (GE) Wash Buffer 1 with Triton X-102, followed by Agilent GE Wash Buffer 2 with Triton X-102. After washing, all slides were immediately scanned at $5 \mu \mathrm{m}$ resolution by using a PerkinElmer Scan Array Express array scanner. The resulting images were quantified by using Agilent's Feature Extraction software. The differentially expressed miRNAs were identified by using Agilent miRNA gene arrays protocol. To increase the reliability of the data, miRNA species with hybridization intensities $<1.5$ times the average hybridization intensity (mean) were excluded from analysis. The miRNA clustering analysis was performed with the Hierarcical clustering algorithm provided in Multi Experiment Viewer, MeV4.0 software package (www.tm4.org/mev.html). After screening the differentiated miRNA, we predicted the targeting genes using targetscan software. Sites with $\mathrm{P}_{\mathrm{CT}}>0.75$ are with higher probability of preferential conservation.

\section{SKM-1 cell line}

Our lab purchased SKM-1 cell line from Health Science Research Resources Bank in Japan, and cultured in Roswel Park Memorial 1640 medium (Gibico Inc.) supplemented with $10 \%$ fetal bovine serum (Gibico Inc.) in a $37^{\circ} \mathrm{C}, 5 \% \mathrm{CO}_{2}$ incubator.

\section{siRNA transfection}

SKM-1 cells in log phase were divided into three groups. One group of cells was planted at a concentration of $5 \times 10^{5}$ cells/well in 96-well plates and transfected with SLC7A5-RNAi (SLC7A5 downregulation group) in serum free medium. Polybrene was added to improve transfection efficiency as enhancing reagent. After 8 hours, the medium was changed for complete medium. The second group of cells was transfected with negative control (negative control group) in the same way as described previously. The third group of cells without intervention served as blank control group. After 3 days of transfection, transfection efficiency was examined using fluorescence microscopy and flow cytometry. Cells were harvested for further experiments.

\section{Western blot analysis}

Approximately $2 \times 106$ cells were lysed in RIPA buffer (50 mM Tris-HCl, pH 8.0; 1 mM EDTA, pH 8.0; 
5 mMDTT; and $2 \%$ SDS) and total protein was extracted and determined with a BCA assay. A 10\% SDS-PAGE gel was loaded with $20 \mu \mathrm{g}$ of total protein. The antibodies, used for Western blot analysis, included rabbit polyclonal anti-SLC7A5 antibody (Cell signaling tech Inc., 1:1000), anti-GAPDH antibody (Kangchen Inc., 1:10000), and HRP-conjugated anti-rabbit secondary antibody (Kangchen Inc., 1:10000).

\section{Cell proliferation assay}

Cell growth was determined by CCK-8 assay (Dojindo, Japan). Briefly, 5000 cells were seeded onto a 96-well plate in quadruplicate for each condition. CCK-8 reagent was added to each well in $10 \mu \mathrm{L}$ after 4 hours after the cells were seeded, and then the cells were incubated for another 5 hours. Each sample was measured at 450nm and $630 \mathrm{~nm}$ for its absorbance value at $0,24,48,72 \mathrm{~h}$ since the incubation.

\section{Cell apoptosis analysis}

Cell apoptosis was determined by Annexin V Apoptosis Detection Kit APC (eBioscience Inc.). Approximately $2 \times 10^{5}$ cells from each experimental group were washed with PBS and then suspended by annexin-binding buffer. Subsequently, $5 \mu \mathrm{L}$ annexin $\mathrm{V}$ and $5 \mu \mathrm{L}$ diluted PI were added to each sample. Incubated for another 30 minutes, the cells were diluted by buffer and then analyzed with a flow cytometry.

\section{Cell cycle analysis}

SKM-1 cells were exposed to various YSY01Aconcentrations for 48h, washed with twice cold PBS and harvested. The cell aggregate then was suspended in $70 \%$ ice-cold ethanol at $-20^{\circ} \mathrm{C}$ overnight. The fixed cells were incubated with RNAase A for $30 \mathrm{~min}$ at $37^{\circ} \mathrm{C}$, and stained with PI (propidiumidodide). The DNA content was detected using flow cytometry.

\section{Electron microscopy}

Electron microscopy was conducted according to thestandard procedures. Approximately 0.25-mm-thick SKM-1 cell slices were postfixed briefly in $2 \%$ osmium tetroxide in $0.1 \mathrm{M}$ cacodylate buffer, dehydrated in graded ethanol and embedded in epoxy resin. Ultrathin 60-70 nm sections were cut using a Leica ultra-microtome, mounted on 200-meshcopper grids. Grids were examined and photographed using a transmission electron microscope (Philips CM200).

\section{Statistical analysis}

All statistical analyses were performed using SPSS 15.0 software. Results for continuous variables were expressed as mean \pm SD. The Kruskal-Wallis $\chi^{2}$-test was used to determine the significance of association between groups or proportions. Student's t-test was used to compare the means of results where appropriate. A value of $\mathrm{P} \leq 0.05$ was considered statistically significant.

\section{ACKNOWLEDGMENT}

This work was supported by grants from China National Natural Science fundation (81070404 and 81100340).

\section{CONFLICTS OF INTEREST}

The authors declare that they have no conflicts of interest.

\section{Authorship contribution}

Yan MA participated in experiment design, performed the majority of the experiments, and wrote the manuscript. Jing Song and Bobin Chen performed statistical analysis of data and reviewed the manuscript. Xiaoping $\mathrm{Xu}$ and Guowei Lin reviewed and supervised research and reviewed and approved the manuscript.

\section{REFERENCES}

1. Steensma DP, Bejar R, Jaiswal S, Lindsley RC, Sekeres MA, Hasserjian RP, Ebert BL. Clonal hematopoiesis of indeterminate potential and its distinction from myelodysplastic syndromes. Blood. 2015; 126:9-16.

2. Radivoyevitch T, Saunthararajah Y. Sex difference in myelodysplastic syndrome survival andbalance in randomized clinical trials. J Clin Oncol. 2014; 32:60-61.

3. Rhyasen GW, Bolanos L, Fang J, Jerez A, Wunderlich M, Rigolino C, Mathews L, Ferrer M, Southall N, Guha R, Keller J, Thomas C, Beverly LJ, et al. Targeting IRAK1 as a therapeutic approach formyelodysplastic syndrome. Cancer Cell. 2013; 24:90-104.

4. Killick SB, Carter C, Culligan D, Dalley C, Das-Gupta E, Drummond M, Enright H, Jones GL, Kell J, Mills J, Mufti G, Parker J, Raj K, et al. Guidelines for the diagnosis and management of adultmyelodysplastic syndromes. Br J Haematol.2013; 164:503-525.

5. Mori F, Sacconi A, Canu V, Ganci F, Novello M, Anelli V, Covello R, Ferraresi V, Muti P, Biagini R, Blandino G, Strano S. miR-181c associates with tumor relapse of high grade osteosarcoma. Oncotarget. 2015; 6:13947-13961. doi: 10.18632/oncotarget.3539.

6. Castro NP, Fedorova-Abrams ND, Merchant AS, Rangel MC, Nagaoka T, Karasawa H, Klauzinska M, Hewitt SM, Biswas K, Sharan SK, Salomon DS. Cripto-1 as a novel therapeutic target for triple negative breast cancer. Oncotarget. 2015; 6:11910-11929. doi: 10.18632/oncotarget.4182. 
7. Stückrath I, Rack B, Janni W, Jäger B, Pantel K, Schwarzenbach H. Aberrant plasma levels of circulating miR-16, miR-107, miR-130a and miR-146a are associated with lymph node metastasis and receptor status of breast cancer patients. Oncotarget. 2015; 6:13387-13401. doi: 10.18632/oncotarget.3874.

8. Cui R, Meng W, Sun HL, Kim T, Ye Z, Fassan M, Jeon YJ, Li B, Vicentini C, PengY, Lee TJ, Luo Z, Liu L, et al. MicroRNA-224 promotes tumor progression in nonsmall cell lung cancer. Proc Natl Acad Sci U S A. 2015; 112:E4288-97. doi: 10.1073/pnas.1502068112.

9. Li Z, Chen P, Su R, Li Y, Hu C, Wang Y, Arnovitz S, He M, Gurbuxani S, Zuo Z, Elkahloun AG, Li S, Weng H, et al. Overexpression and knockout of miR-126 both promote leukemogenesis through targeting distinct gene signaling. Blood. 2015; 126:2005-2015.

10. Yan W, Xu L, Sun Z, Lin Y, Zhang W, Chen J, Hu S, Shen B. MicroRNA biomarker identification for pediatric acute myeloid leukemia based on a novel bioinformatics model. Oncotarget. 2015; 6:26424-36. doi: 10.18632/ oncotarget. 4459 .

11. Kotagama K, Chang Y, Mangone M. miRNAs as Biomarkers in Chronic Myelogenous Leukemia. Drug Dev Res. 2015; doi: 10.1002/ddr.21266.

12. Emmrich S, Engeland F, El-Khatib $M$, Henke $\mathrm{K}$, Obulkasim A, Schöning J, Katsman-Kuipers JE, Michel Zwaan C, Pich A, Stary J, Baruchel A, de Haas V, Reinhardt D, et al. miR-139-5p controls translation in myeloid leukemia through EIF4G2. Oncogene. 2015; doi: 10.1038/onc.2015.247.

13. Pons A, Nomdedeu B, Navarro A, Gaya A, Gel B, Diaz T, Valera S, Rozman M, Belkaid M, Montserrat E, Monzo M. Hematopoiesis-related microRNA expression in myelodysplastic syndromes. Leuk Lymphoma. 2009; 50:1854-1859.

14. Hussein K, Theophile K, Büsche G, Schlegelberger B, Göhring G, Kreipe H, Bock O. Aberrant microRNA expression pattern in myelodysplastic bone marrow cells. Leuk Res.2010; 34:1169-1174.

15. Choi JS, Nam MH, Yoon SY, Kang SH. MicroRNA194-5p could serve as a diagnostic and prognostic biomarker in myelodysplastic syndromes. Leuk Res. 2015; 39:763-768.

16. Wang L, Song J, Zhang J, Zhu C, Ma Y, Xu X. Lentiviral vector-mediate ATG3 overexpression inhibits growth and promotes apoptosis of human SKM-1 cells. Mol Biol Rep. 2014; 41:2093-2099.

17. Ma Y, Chen B, Xu X, Lin G. Prospective nested case-control study of feature genes related to leukemic evolution of myelodysplastic syndrome. Mol Biol Rep. 2011; 40:469-476.

18. Geier EG, Schlessinger A, Fan H, Gable JE, Irwin JJ, Sali A, Giacomini KM. Structure-based ligand discovery for the Large-neutral Amino Acid Transporter 1, LAT-1. Proc Natl Acad Sci U S A. 2013; 110:5480-5485.

19. Hayashi K, Jutabha P, Endou H, Sagara H, Anzai N. LAT1 is a critical transporter of essential amino acids for immune reactions in activated human T cells. J Immunol. 2013; 191:4080-4085.

20. Fukumoto S, Hanazono K, Fu DR, Endo Y, Kadosawa T, Iwano H, Uchide T. A new treatment for human malignant melanoma targeting L-type amino acid transporter 1 (LAT1): a pilot study in a canine model. Biochem Biophys Res Commun. 2013; 439:103-108.

21. Ichinoe M, Mikami T, Yoshida T, Igawa I, Tsuruta T, Nakada N, Anzai N, Suzuki Y, Endou H, Okayasu I. High expression of L-type amino-acid transporter 1(LAT1) in gastric carcinomas: comparison with non-cancerous lesions. Pathol Int, 2011; 61:281-289.

22. Rosilio $C$, Nebout $M$, Imbert $V$, Griessinger $E$, Neffati Z, Benadiba J, Hagenbeek T, Spits H, Reverso J, Ambrosetti D, Michiels JF, Bailly-Maitre B, Endou H, et al. L-type amino-acid transporter 1 (LAT1): a therapeutic target supporting growth and survival of T-cell lymphoblastic lymphoma/T-cell acute lymphoblastic leukemia. Leukemia, 2015; 29:1253-1266.

23. Imai H, Kaira K, Oriuchi N, Yanagitani N, Sunaga N, Ishizuka T, Kanai Y, Endou H, Nakajima T, Mori M. L-type amino acid transporter 1 expression is a prognostic marker in patients with surgically resected stage I non-small cell lung cancer. Histopathology. 2009; 54:804-813. 